\title{
FURTHER OBSERVATIONS ON HYPERENDEMIC ONCHOCERCIASIS IN THE UPPER TARABA RIVER VALLEY, NIGERIA
}

AKOGUN O. B.* \& RENZ A.**

Keywords : Onchocerca volvulus, blinding onchocerciasis. Taraba river valley. Nigeria.

Collowing an earlier report of severe ocular onchocerciaS sis in the middle Taraba river valley, Nigeria (Akogun 1992) and the need to identify all communities which may benefit from ivermectin treatment, an epidemiological survey of the less easily accessible communities of the upper Taraba river valley was carried out.

Of the 2.176 people from 12 communities that were examined for the presence of the microfilariae of Onchocerca volvulus and the clinical symptoms of onchocerciasis, $61.8 \%$ were microfilarial positive and the intensity of infection was 78.9 microfilariae/skin-snip. Infection rates exceeded $60 \%$ in nine communities (with $100 \%$ in one community and $98.5 \%$ in another). Two communities had $45.4 \%$ and $32.2 \%$ while the remaining community had $9.1 \%$. As the rate and intensity of infection increases so does the prevalence of leopard skin and nodule carriage.

Eye lesions and blindness were broadly defined as any visible circular or localised corneal 'snowflakes' or occlusion of the pupil and inability to read the 6/60 ' $\mathrm{E}$ ' card at $3 \mathrm{~m}$ respectively. Eye lesions were especially high $(27.1 \%)$ and reached $56.7 \%$ in one community. In six communities the proportion of the population with eye lesions exceeded $30 \%$. Blindness as recorded in all but one community and its prevalence ranged between $3.2 \%$ and $62.5 \%$ with an overall average of $19.7 \%$. Eye lesions were rare before the age of 20 years but blindness increased from $17.4 \%$ in age 20 - 29 years to $54.6 \%$ at age 50 years $(\mathrm{N}=972)$. These surveys indicate that the upper Taraba valley is worse than the mid-valley area and that the Taraba has the worst blinding onchocerciasis in Nigeria.

The transmission of $O$. volvulus at three sites of the Taraba valley is currently been assessed.

\footnotetext{
* Federal University of Technology, Department of Biological Sciences, Yola, Nigeria

**Universität Hohenheim, Fachgebiet Parasitologie (Institut für

Zoologie), 70599 Stuttgart, Germany
} 